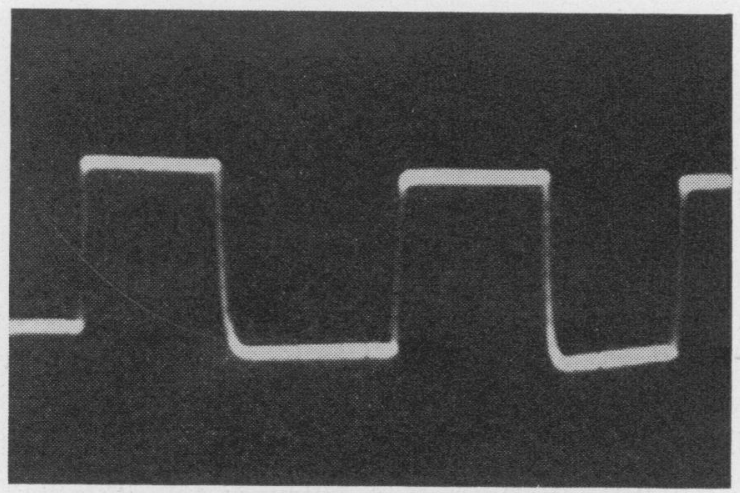

Fig. 5-Square-wave output for $100 \mathrm{kc}$ pulses by the input $R C$ circuit. These pulses trigger the tube "on" and "off," as in the circuit described earlier. When the tube is triggered "off," the $0.1-\mu \mathrm{f}$ capacitor is charged exponentially through the plate resistor (250,000 ohms). When the tube is triggered "on," the output capacitor discharges exponentially through the tube.

Fig. 5 shows the output wave form when the tube is triggered at $100 \mathrm{kc}$. The leading edge has a rise time of approximately 0.2 microsecond. The full capabilities of this tube have not yet been determined; however, it is believed that it can be made to trigger at frequencies as high as $1 \mathrm{Mc}$ since no gas-deionization time is involved.

\title{
Electronic Techniques Applied to Analogue Methods of Computation*
}

\author{
G. D. McCANN†, C. H. WILTS $\dagger$, AND B. N. LOCANTHI $\dagger$, ASSOCLATE, IRE
}

Summary-This paper describes in detail the electronic devices and principles that have been developed for the California Institute of Technology (CIT) electric analogue computer. This is a generalpurpose, large-scale computer applicable to a wide range of linear and nonlinear ordinary algebraic or differential equations and linear and nonlinear partial differential equations.

In addition to the basic principles of the computer, a detailed discussion is given of those elements considered to be of particular interest. These include the devices for generating the arbitrary functions of the independent variable (the excitation functions), the amplifiers for producing active linear elements such as negative impedances and for representing the nonsymmetrical terms of the matrix specifying the differential equations, the multipliers for producing arbitrary functions of the dependent variables (nonlinear elements).

Performance data on these devices are presented, together with analogies and solutions of representative types of problems.

\section{INTRODUCTION}

$\mathrm{T}$ HE ELECTRIC analogue computer, which has been developed by the California Institute of Technology provides a device having as wide a field of application as is considered practical with the electric analogue principle. It is thought that the greatest field of usefulness of such a computer lies in the accuracy range that is limited to the order of 1 per cent, which covers the vast majority of problems in the general field of engineering analysis. With this accuracylimitation, great simplification in the computer and its operation results from the use wherever possible of $R$, $L, C$, and transformer circuits in simulating the linear

* Decimal classification: 621.375 .2 . Original manuscript received by the Institute, October 8,1948 ; revised manuscript received, February 24, 1949. Presented, 1948 IRE West Coast Convention, Los Angeles, Calif., September 30, 1948.

† California Institute of Technology, Pasadena, Calif. terms of both algebraic and differential equations. ${ }^{1,2}$ This is in contrast to computers which employ electronic amplifiers for all integration or differentiation. They provide higher accuracy, but are restricted in their field of application as compared to that practicable with a computer of the type discussed here.

Electric analogue computers are limited to one independent variable that can be continuously represented. However, by the use of finite-difference methods, such as must be employed for digital computers, more independent variables can be handled. The CIT computer is designed to handle partial differential equations with up to three independent variables. Two basic circuit techniques are employed. For the first of these, one independent variable is continuously represented as time on the computer. ${ }^{1,2}$ In the other, all independent variables are represented by finite-difference methods, in which case the analogous circuit becomes a steady-state ac or dc mesh. ${ }^{3}$ Both techniques are applicable to both ordinary and partial differential equations. It is the application of the first technique that requires most of the electronic equipment, such as devices to generate suitable functions of the independent and dependent variables, multipliers for multiplying any two variables together, and amplifiers to provide the proper poweroutput levels from these devices and to produce negative impedance terms and the unilateral or unsymmetrical terms of a matrix or servosystem.

${ }^{1}$ G. D. McCann, "The mechanical transients analyzer," Proc. $N E C$, vol. 2, p. $372,1946$.

${ }^{2}$ E. L. Harder and G. D. McCann, "A large scale general-purpose electric analog computer," AIEE Technical Paper 48-112.

${ }^{3}$ G. D. McCann and C. H. Wilts, "Application of electric analog computers," Jour. Appl. Mech., 1949. 


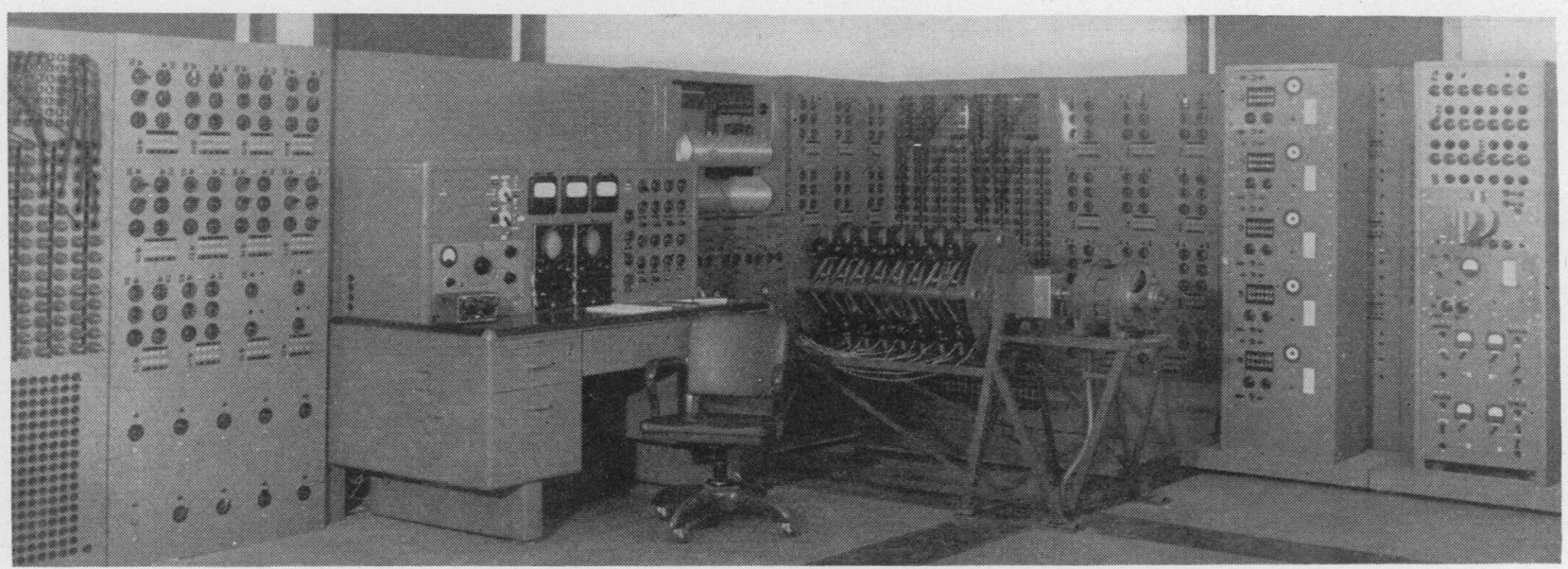

Fig. 1-A general view of the California Institute of Technology computer.

It is these recently developed basic electronic elements of the computer that are to be described in this paper. The general arrangement of the computer, a photograph of which is shown in Fig. 1, has been described in a previous paper. ${ }^{2}$

\section{Examples of Computer Techniques}

The required specifications for the analogue elements is best considered by first illustrating their application to specific problems and describing the analogue techniques employed. The basic analogue elements employed in the computer are listed in Fig. 2 in terms of their symbolization as employed for circuit layouts. Typical analogous circuits for ordinary differential equations are given in Figs. 3 and 4.

As illustrated in the examples, $R, L$, and $C$ circuit elements are usually employed for all linear "self-impedance" terms. They are also employed for bilateral mutual terms that can be formed with junction points between the meshes simulating the simultaneous equations and for transfer functions. Special transformers ${ }^{2}$ are used wherever possible for bilateral mutuals requiring circuit isolation or, in conjunction with amplifiers, to provide a four-terminal unilateral element.
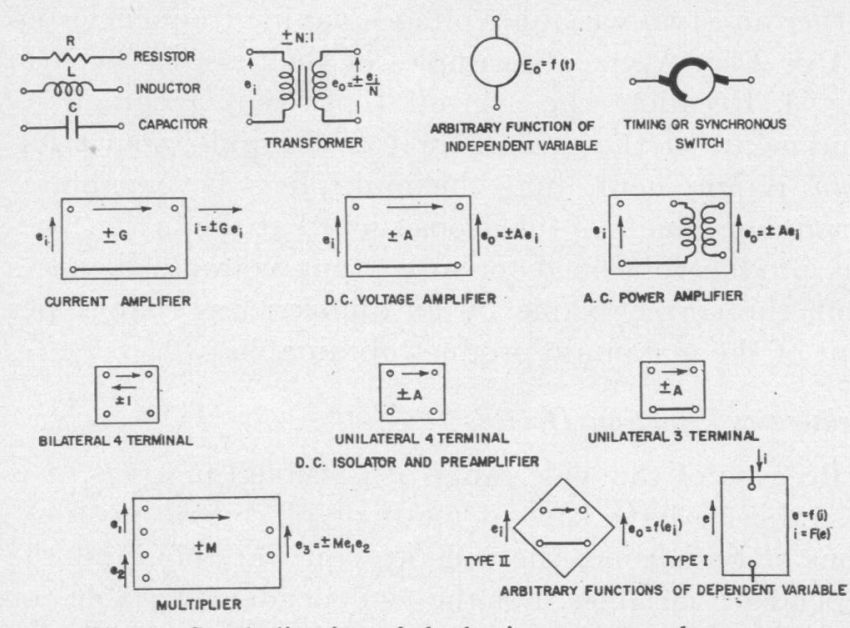

Fig. 2-Symbolization of the basic computer elements.
The use of such circuit elements fixes the impedance level and the rate of computation. To minimize stray coupling and stray pickup problems and enable the quick setup of a wide range of circuits, the circuit impedance base must be kept low and the power level reasonably high. Unintentional impedances, such as the output impedance of a voltage amplifier, must be kept below a few ohms if they are series elements, and above several hundred thousand ohms (and in extreme cases, 10 or 20 megohms) if they are shunt impedances. In order that the elements are sufficiently close to being ideal inductors, etc., the natural frequencies of the analogue circuits must lie within certain limits. A normal operating frequency range of from $10 \mathrm{cps}$ to 1 or 2 kc was chosen. ${ }^{2}$ However, because of this wide frequency range, transformers have certain limitations. It is diffcult to keep the ratio of the series to magnetizing impedance of a transformer sufficiently low over such a wide operating frequency range. This, together with

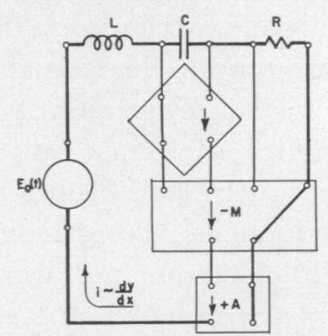

Fig. 3-Illustrative analogy for an ordinary differential equation:

$$
F_{0}(x)=k_{1} \frac{d^{2} y}{d x^{2}}+f_{1}(y) \frac{d y}{d x}+k_{2} y .
$$

Analogous circuit equation:

$$
E_{0}(t)=L \frac{d^{2} q}{d t^{\prime 2}}+\frac{A M R}{C} F_{1}(q) \cdot \frac{d q}{d t^{\prime}}+\frac{q}{C}\left\{R \frac{d q}{d t^{\prime}} \text { negligible }\right\} .
$$

Numerical conversion formula:

$$
y=a \frac{F_{0}}{E_{0}} q ; \quad L=\frac{a}{n^{2}} k_{1} ; \quad \frac{I}{C}=a k_{2} ; \quad \frac{A M R}{C} \cdot F_{1}(q)=\frac{a}{n} f_{1}(q)
$$

where

$a=$ impedance-base change factor

$n=$ independent-variable conversion factor.

(One unit of $x$ in its system of units equals $1 / n$ units of $t^{\prime}$ seconds. 


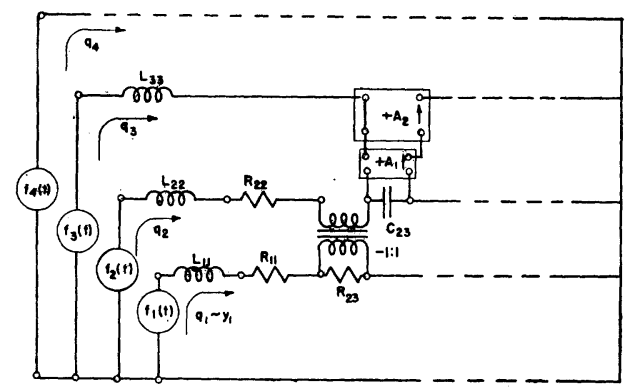

Fig. 4-Illustrative analogy for simultaneous equations:

$$
\begin{aligned}
& f_{1}(t)=K_{11} \frac{d^{2} y_{1}}{d t^{2}}+k_{11} \frac{d y_{1}}{d t}+k_{12} \frac{d y_{2}}{d t}+\cdots \\
& f_{2}(t)=K_{22} \frac{d^{2} y_{2}}{d t^{2}}+k_{22} \frac{d y_{2}}{d t}+k_{12} \frac{d y_{1}}{d t}+\cdots \\
& f_{3}(t)=K_{33} \frac{d^{2} y_{3}}{d t^{2}}+k_{23} y_{2}+\cdots \\
& f_{4}(t)=\cdots .
\end{aligned}
$$

the tendency of transformer-coupled feedback circuits to "motorboat," constitute the major difficulties sometimes encountered in the use of transformers. It is, therefore, occasionally necessary to use four-terminal "isolators" which operate properly from zero frequency (dc) to the upper limit of 1 or $2 \mathrm{kc}$ (see Fig. 4). The general philosophy regarding accuracy has been to achieve 1 per cent accuracy in all elements. Thus, an inductor should exhibit a variation in effective inductance which is less than 1 per cent of its value throughout the range in operating level and operating frequency (in this case, 100 to $1,000 \mathrm{cps}$ ). In addition, its effective resistance should be less than 1 per cent of its reactance at all frequencies and levels (i.e., its $Q$ should be greater than 100). Similarly, all of the electronic equipment must have less than 1 per cent amplitude distortion throughout the operating range. Furthermore, the frequency response of the equipment must not vary more than $0.1 \mathrm{db}$ throughout the frequency range of the computer. It was considered satisfactory if the phase shift were less than $1^{\circ}$ throughout the frequency range. However, since these devices are frequently used in cascade and in feedback circuits, the phase and amplitude characteristics must be controlled to eliminate oscillations at high and low frequencies. To prevent low-frequency "motorboating," all electronic equipment except the arbitrary function of the independent-variable (forcingfunction) generators was designed for operation from zero frequency $(\mathrm{dc})$ to the upper frequency limit of the computer. To simplify elimination of high-frequency oscillations, it was found desirable to extend the highfrequency limit of all equipment as far as practicable.

\section{Power Amplifiers}

Three types of amplifiers are shown in Fig. 2-ac power amplifiers, dc current generators, and dc voltage amplifiers. Although they are frequently used for other purposes, their most common uses are: (1) Ac power amplifier: to amplify the output of the forcing-function generators (arbitrary function of the independent vari- able) and provide a low source impedance. (2) Dc current generator: to produce a current as a "forcing function." (3) Dc voltage amplifier - to produce negative or unilateral impedances, and to amplify the output of generators of an arbitrary function of the dependent variable. Typical examples of the use of the dc amplifiers are given in Figs. 3 and 4. The dc amplifier input impedances must be kept high so that they will not distort the circuit across which they are to be connected. However, since they usually can be used with a small input signal, the circuits can be so set up that 500,000 ohms is a sufficiently high input impedance. For most problems, power levels corresponding to a maximum current of 30 to $50 \mathrm{ma}$ and a voltage of 50 to 100 volts are adequate.

Two types of dc voltage amplifiers were found desirable. One of these has a negative or $180^{\circ}$ phase-reversal characteristic, and is to be used particularly for servomechanism problems. It has a maximum gain of 50 and is usually used in groups of three or more. The other is a positive-gain amplifier. For this a gain of 100 is usually adequate when it is used to produce a negative or unilateral impedance, since the accuracy limitation of the computer is 1 per cent. However, in limited cases, larger gains are required. In order to provide for this additional gain, the amplifier can be used with a preamplifier, which has a gain variable from 0 to 10 . It is considered adequate if the current amplifiers can deliver about $50 \mathrm{ma}$ and 100 volts. Their effective source impedance should be a megohm or more.

As can be seen from the illustrative circuits (Figs. 3 and 4), it is necessary that all analogue elements be capable of operation with no connection between normal ground terminal and true system ground, or they must be provided with isolating elements at their terminals. As will be discussed later, it was found practicable to design the electronic equipment for operation with no connection to system ground, thereby affording considerable simplification.

\section{Multipliers}

The multipliers must be capable of multiplying together any two variable voltages having frequencies up ti 1 or $2 \mathrm{kc}$. Atypical examples of their use is given in Fig. 3. Recalling the over-all 1 per cent accuracy requirements of the computer, it is desirable, as a minimum requirement, that the multipliers be capable of forming products of functions such that the instantaneous product obtained for any input voltages deviates from the correct value by an amount less than 1 per cent of the maximum product obtainable.

\section{Arbitrary Function Devices}

Because of the wide range of possible functions, it is desirable to have several methods of generating functions of both time, the independent variable, and the dependent variables. For the "independent variable" or time functions, six square-wave voltage generators, con- 
sisting of storage-battery circuits, and six variable-frequency sine-wave generators are provided. These are described in footnote reference 2 . In addition, however, it is necessary to be able to generate any time function that can be plotted or expressed mathematically. Many important nonlinear functions of the dependent variable, such as saturation curves, square-law characteristics, sudden discontinuities, etc., can be generated with ${ }^{*}$ relatively simple diode circuits. In addition, however, provision must be made for perfectly arbitrary functions.

\section{Description of Electronic Components}

\section{Generator of Arbitrary Functions of the Independent Variable}

Since the computer operates in the audio-frequency range, it is natural to consider the possible application of the various principles employed for sound recording, including the light-beam-photocell principle, magnetic tape, or wire recording and conventional disk recording and playback techniques. All of these methods were tried experimentally and found practical. As will be discussed later, the light-beam-photocell principle could be most easily and practically developed into a form suitable to the needs of the computer, and was adopted for one form of "forcing-function" generator. However, the use of any of the above principles leads to one practical difficulty. If the function of the independent variable is unknown and the only known data are some actual solutions obtained from experimental tests, it is desirable to have a generator in which the arbitrary function can be varied until, by trial and error, a correct solution is obtained.

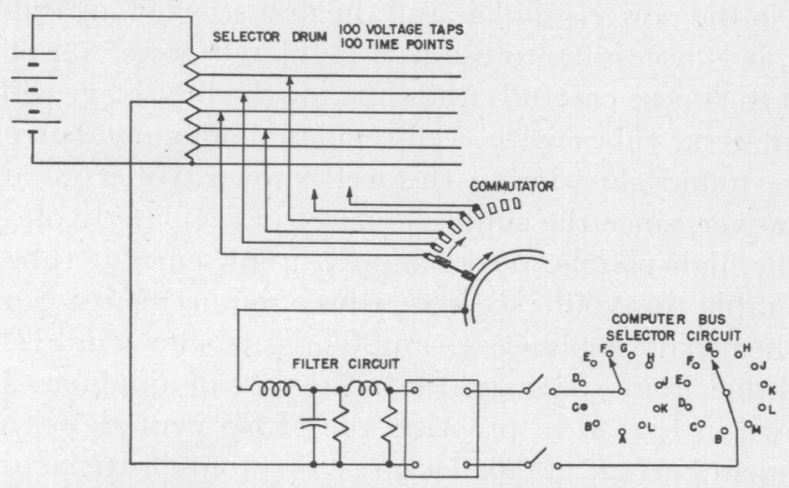

Fig. 5-Schematic diagram of the arbitrary forcing-function generator Type I; variable gain amplifier, 1-ohm output impedance, 50 volts at 1 -ampere output.

\section{Type I}

The device illustrated in Fig. 5, although limited in the maximum frequency which it can represent, does provide for rapid changes in the forcing function. As shown in the schematic diagram (Fig. 5), 100 voltage taps are provided which may be selected by the adjustment of 100 switches, each of which connect to a segment of a commutator, thus providing 100 points in time. A filter circuit provides for a smooth transition between points. The 100 voltage taps are rods placed around the periphery of a circular drum, over which are 100 circular rings each connecting to a segment of a mechanical commutator. Adjustment of the function generator is accomplished by rotating the rings around the drum. Three of these devices are provided in the computer. They may be used separately, or two may be ganged in series to provide up to 200 points in time.

\section{Type II}

The generator employing the photoelectric principle is illustrated in Fig. 6. A photographic film is produced
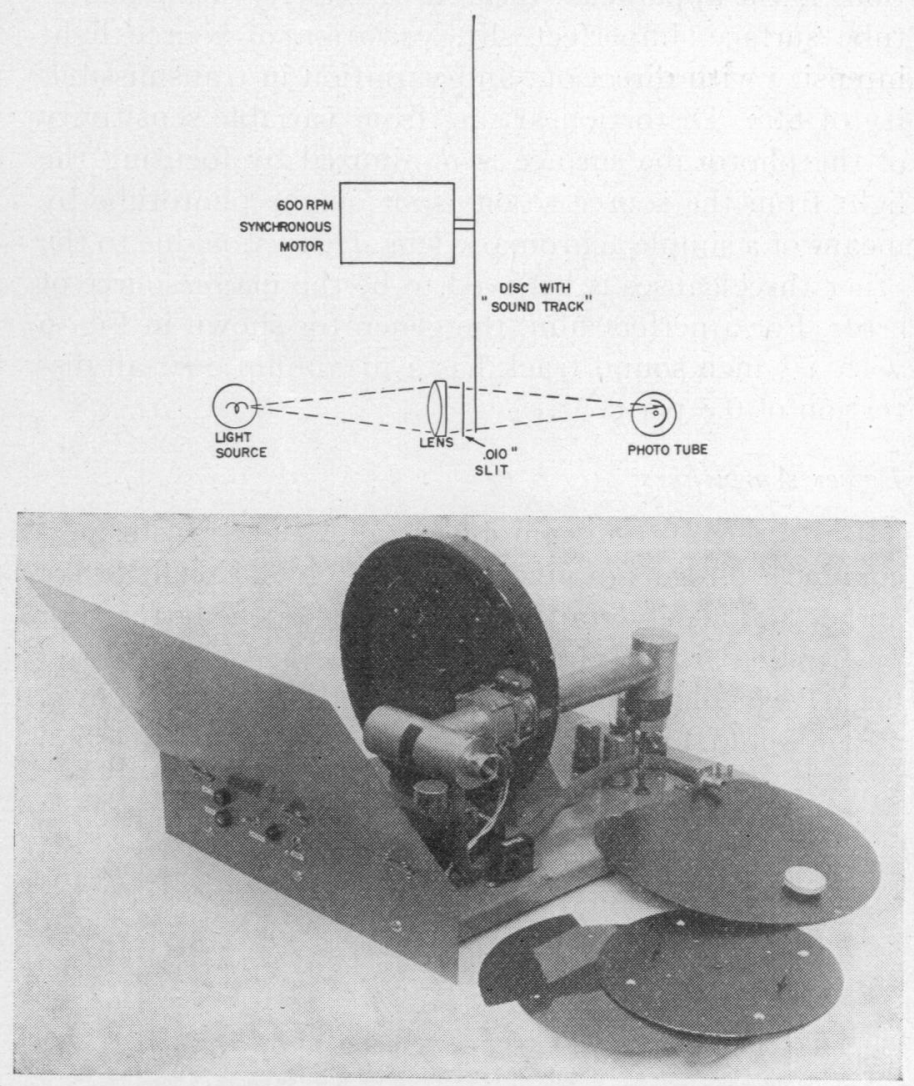

Fig. 6-(a) Schematic diagram of the generator of an arbitrary function of independent variable. (b) Photograph of a generator of an arbitrary function of independent variable.

with a circular "sound track" having a variable width proportional to the desired function. This film and a narrow slit are interposed between a light source and a phototube. When the film is rotated at synchronous speed (10 rps), a voltage is produced which is a periodic function of time, and which is, during each cycle, proportional to the desired function of the independent variable. The film diameter is 11 inches, with provision for a maximum track width of $\frac{5}{8}$ inch. A simple ac amplifier is used to increase the signal level to about 1 volt.

There are six principal sources of distortion in such an optical-photoelectric system. Two of these result from geometrical properties of the system. One arises if the width of the "sound track" is made exactly propor- 
tional to the desired function. In this case, the angle subtended by the track is not exactly proportional to the desired function, but is related by a simple trigonometric expression which departs appreciably from linearity if the angle becomes too large. Since the light transmitted is proportional to the angle, a lack of linearity results. The other distortion is a frequency-sensitive amplitude distortion. It arises because of the finite slit width and limits the detail possible because of the averaging effect over the width of the slit. These distortions were made less than 0.1 per cent by maintaining suitable ratios of critical dimensions.

Four other sources of distortion are due to imperfections in the apparatus: variable sensitivity of the phototube surface, imperfect slit, variation of source light intensity with direction, and variation in transmissibility of film. Distortion arising from variable sensitivity of the phototube surface is minimized by focusing the light from the source to one spot on the phototube by means of a simple achromatic lens. Distortion due to the other three causes is believed to be the major source of error. For a perfect film, the generator shown in Fig. 6 with a $\frac{5}{8}$-inch sound track has a maximum over-all distortion of 0.3 per cent.

\section{Power Amplifiers}

Both the current amplifiers and the transformercoupled voltage amplifiers are of conventional design and do not merit detailed discussion. For the latter, four push-pull-parallel 6L6's with approximately $60 \mathrm{db}$ of negative feedback met the special output requirement of 50 volts and 1 ampere with an internal impedance of 1

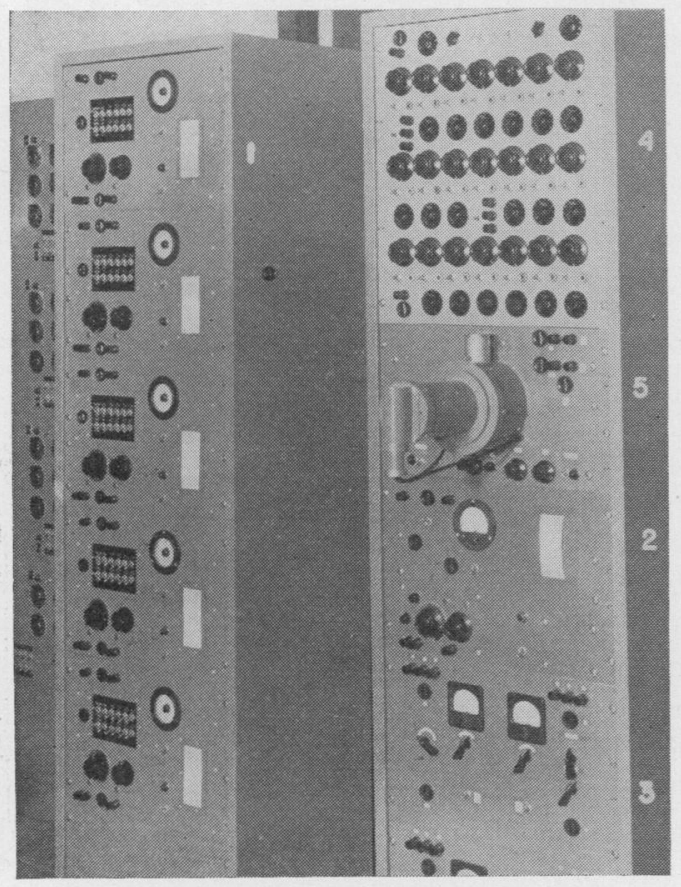

Fig. 7-Photograph of amplifiers and arbitrary-function generators.

(1) Negative gain servo amplifiers. (2) Positive-gain dc amplifier. (3) Voltage and current limiters. (4) Dependent-variable function generator-Type I. (5) Dependent-variable function generatorType II.

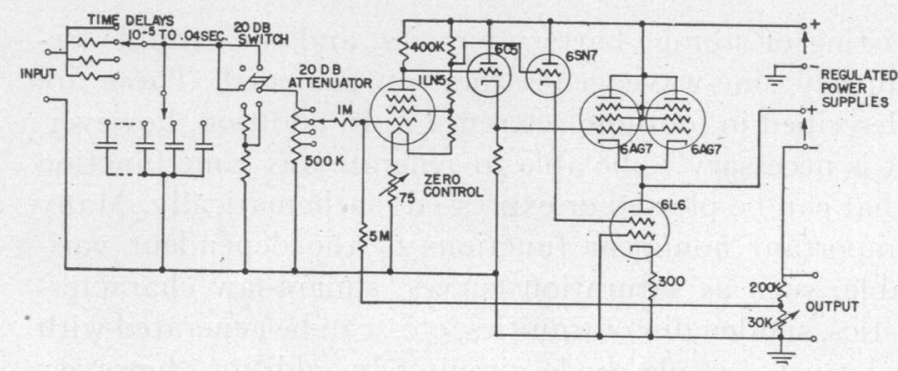

(a)

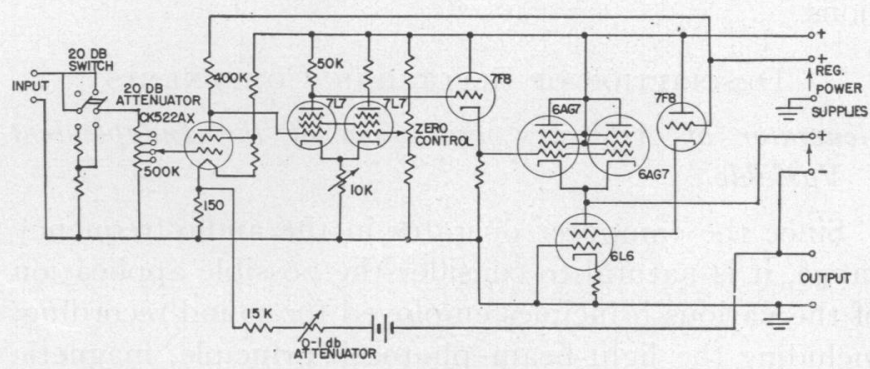

(b)

Fig. 8-Schematic diagrams of dc amplifiers. (a) Servo or negativegain amplifier. (b) Positive-gain amplifier.

$\mathrm{ohm}$. The amplification varies less than $0.1 \mathrm{db}$, and the phase shift is considerably less than $1^{\circ}$ from 10 to 2,000 cps.

The dc voltage amplifiers presented the most difficult problems. It is of great importance to minimize the drift in output voltage. In typical servo problems, amplifiers may be cascaded with an over-all gain of many thousand, so that drifts of even a few millivolts at the input may be objectionable. After considerable experimental and theoretical work on the matter, satisfactory amplifiers were developed. Since the main source of drift lies in the power supplies and the first stage of an amplifier, it is necessary to use well-regulated power supplies and to choose carefully the tube for the first stage. Filament-type tubes with well-regulated filament current were found superior to the well-known Miller circuit. However, since the simplest source of regulated voltage is the high-voltage power supply, a low-current tube is desirable to avoid excessive power requirements. Some of the negative-gain servo amplifiers are shown in Fig. 7, and the circuit diagrams for both types of amplifiers are shown in Fig. 8. In practice, the 1LN5 pentode with a filament current of 0.040 ampere was found satisfactory for the negative-gain amplifier. A subminiature hearingaid tube, the CK522AX, is used for the positive-gain amplifier, since its filament current is only $0.020 \mathrm{am}$ pere, while its drift and microphonism characteristics are very satisfactory. This tube cannot be used for the negative-gain amplifier because of its inability to provide a sufficiently large output voltage in a one-stage amplifier.

After about a two-hour warm-up period, the drift as referred to the grid of the first tube settles down to a value that does not exceed 1 millivolt per hour. A typical drift record is shown in Fig. 9. The sharp pips 


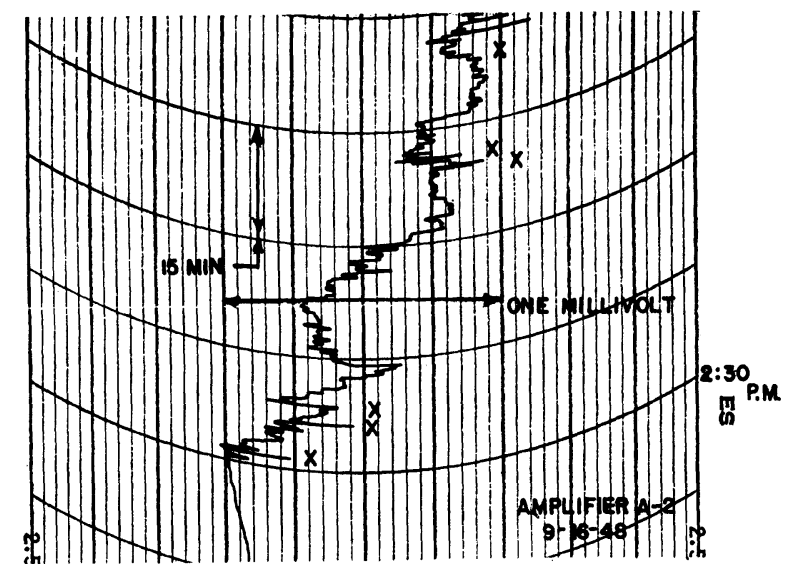

Fig. 9-Typical drift characteristic of a negative-gain amplifier.

marked by $X$ were due to supply-line switching surges. Since this drift stability exceeds the computer requirements, no attempt was made to obtain further improvement.

The other major problems in the design of the dc amplifiers arise because the normal ground terminal $(B-)$ of the amplifier cannot be connected to system ground unless isolators are used. It is conceivable that the analogue circuit impedance to ground may be as high as several thousand ohms, and that the voltage to ground may be as large as 30 or 50 volts. Two undesirable effects can arise. Unless the grid of the input tube of the amplifier is very well shielded from true ground by the $B$ - bus, the capacitance between grid and ground, in series with the normal high impedance from grid to $B-$, results in a grid signal if a voltage exists between $B-$ and true ground. It was considered desirable to have all amplifier chassis connected to true ground, so that shielding was required within the chassis. Without resorting to complete electrostatic shields, the resulting transfer characteristics for the positive-gain amplifier gave rise to an output voltage equal to approximately 0.1 per cent of the signal between $B-$ and true ground. This was considered satisfactory.

The other effect, a 60 -cps ripple appearing on the $B-$ terminal if an impedance is connected between $B$ - and true ground, is more difficult to eliminate. This ripple normally arises from unbalanced capacitance between the ends of the high-voltage winding and the primary winding, as shown in Fig. 10(a). With normal "balancedwinding" procedures, a ripple of about 0.1 volt appears if the impedance $Z$ (Fig. 10) is made equal to 1,000

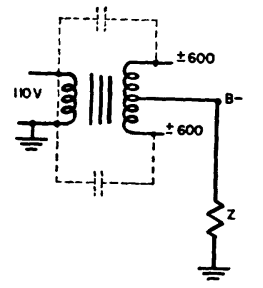

(a)

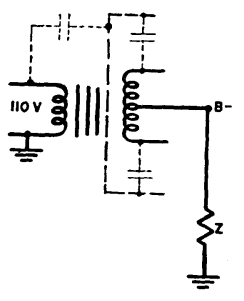

(b)

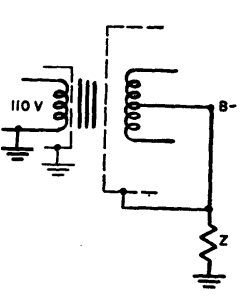

(c)
Fig. 10-Shielding requirements for power-supply transformers. ohms. This corresponds to an unbalanced capacitance of a few hundred $\mu \mu f$. If a single shield, as shown in Fig. 10 (b), is used, conditions are not appreciably improved. If, for example, the shield is connected to true ground, the unbalanced capacitances to the shield still exist. If the shield is connected to $B$-, then the capacitance between primary winding and shield still gives rise to an objectionable signal in the output. By careful choice of connections the ripple voltage may be reduced to 40 or 50 millivolts, but this is much larger than is permissible.

A very satisfactory solution results if two complete electrostatic shields are provided, as shown in Fig. (10(c). Because of construction difficulties, perfect shielding is not economically practicable. However, commercial manufacturers have provided transformers in which the unbalanced leakage capacitance is less than $0.05 \mu \mu \mathrm{f}$ with an increase in cost ranging only from 50 to 100 per cent. This unbalanced capacitance gives rise to a ripple signal of about 0.1 millivolt per thousand ohms impedance.

The other characteristics of the amplifier are achieved by conventional means. The required low output impedance is achieved by use of a cathode-follower output and considerable negative feedback. The available output current is increased substantially above the value for a conventional cathode follower through use of a pentode as cathode impedance. The important characteristics of the amplifiers are listed in Table I.

TABLE I

Performance Data of DC Voltage Amplifiers

\begin{tabular}{|c|c|c|}
\hline & $\begin{array}{l}\text { Negative-Gain } \\
\text { Amplifier }\end{array}$ & $\begin{array}{l}\text { Positive-Gain } \\
\text { Amplifier }\end{array}$ \\
\hline Gain & $\begin{array}{l}0.5 \text { to } 50 \text { in. } 1-\mathrm{db} \\
\text { steps }\end{array}$ & $\begin{array}{l}1 \text { to } 100 \text { continuously } \\
\text { variable }\end{array}$ \\
\hline $\begin{array}{l}\text { Maximum voltage out- } \\
\text { put } \\
\text { Maximum current out- }\end{array}$ & \pm 125 volts peak & \pm 125 . volts peak \\
\hline $\begin{array}{l}\text { put } \\
\text { Input impedance } \\
\text { Output impedance } \\
\text { Ripple level } \\
\text { Stability to line voltage }\end{array}$ & $\begin{array}{l} \pm 65 \text { ma peak } \\
400,000 \text { ohms } \\
25 \text { ohms } \\
1 \mathrm{mv} \mathrm{rms}\end{array}$ & $\begin{array}{l} \pm 65 \text { ma peak } \\
500,000 \text { ohms } \\
0.9 \text { ohms } \\
1 \mathrm{mv} \mathrm{rms}\end{array}$ \\
\hline $\begin{array}{l}\text { fluctuations* } \\
\text { Drift characteristics* } \\
60 \text { cps ripple across } 1,000 \\
\text { ohms between B- and }\end{array}$ & $\begin{array}{l}7 \mathrm{mv} / 10 \text { volts } \\
1 \mathrm{mv} / \text { hour** }\end{array}$ & $\begin{array}{l}0.04 \mathrm{mv} / 10 \text { volts } \\
1 \mathrm{mv} / \text { hour** }\end{array}$ \\
\hline true ground & $1 \mathrm{mv}$ & $0.1 \mathrm{mv}$ \\
\hline
\end{tabular}

* Voltage referred to grid of input tube.

** Typical values after 1-or 2-hour warm-up period.

\section{Isolator and Preamplifier}

Since isolators must, in general, operate with no connection to system ground, power transformers for such devices require the elaborate shielding discussed previously. Thus it is desirable for such isolators to operate without 60 -cps ac power supplies, if convenient. On the other hand, the signal voltage available is frequently small, so that a preamplifier may be desirable to minimize the noise-to-signal ratio of the isolator.

Through the use of an amplitude-modulated carrier device, a very simple isolator results, with negligibly 


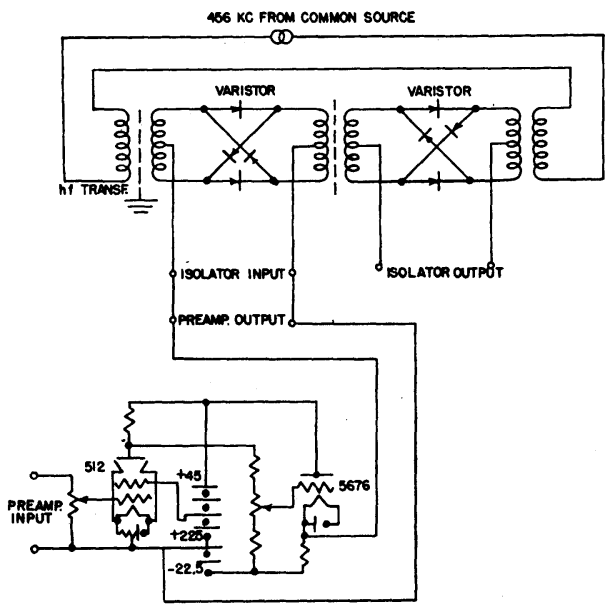

Fig. 11-Schematic diagram of a preamplifier and dc isolator. (NoTE:- Without the preamplifier, the isolator is bilateral in action.)

small capacitance coupling from input to output. Varistor bridges, used as balanced modulators as shown in Fig. 11, have given compact isolators with no 60 -cps power supplies. The design of such a unit is not as straightforward as might be supposed, since the phaseshift requirement for the detected output carries over into a similar requirement regarding relative phase shifts of carrier and sidebands. However, through the use of nonresonant circuits, the phase-shift performance of the isolators can be made satisfactory.

A simple battery-operated amplifier is also shown in the circuit of Fig. 11. Both of these units can be mounted on a very small chassis. The isolator produces a useful signal of about 0.7 volt peak-to-peak and has satisfactory linearity and negligible phase shift in the range from zero frequency (dc) to $1,000 \mathrm{cps}$.

\section{Generator of an Arbitrary Function of the Dependent Variable}

A somewhat more difficult problem is to produce an arbitrary function of the dependent variable. As has been pointed out earlier, the dependent variable can be obtained as a voltage existing at some point in the analogue circuit. The problem is, then, to produce a voltage which is an arbitrary function of another voltage. Two devices have been developed for this purpose.
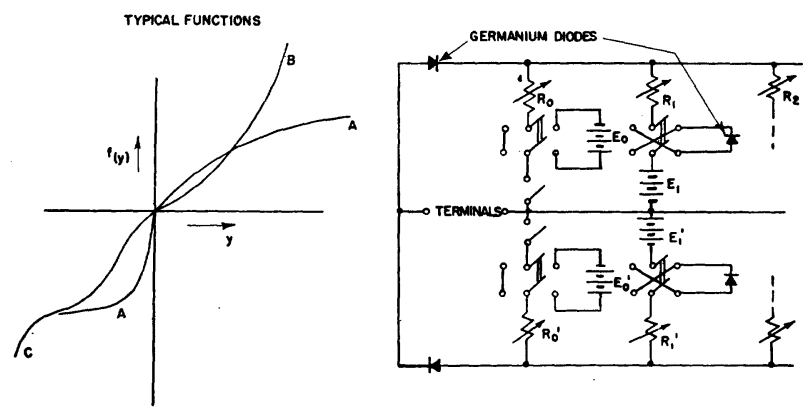

Fig. 12-Schematic diagram of a generator for the arbitrary function of a dependent variable-Type I.

One of the generators (Type I) utilizes crystal diodes and batteries in a manner shown in Fig. 12. The adjusta- ble resistors are made relatively large compared to the forward impedance of the crystals, so that the variations of the latter with current do not affect the over-all impedance appreciably. If the switches are all thrown to the left in Fig. 12, each parallel branch will not conduct current until the terminal voltage rises above the battery voltage of that branch. The resulting current versus voltage characteristic is composed of a series of straight lines, with the slope of successive segments decreasing as the voltage rises. Such a characteristic is shown in curve A of Fig. 12.

If the switches are thrown to the right, a different characteristic results. Each branch produces a circulating current which passes through $R_{0}$. The battery voltage $E_{0}$ is provided to give zero terminal voltage when no external current enters the terminals. As voltage is applied, the current which flows is determined by the effective resistance of all branches in parallel. As soon as the terminal voltage rises above the lowest branch battery voltage, the current in that branch ceases to flow, and the effective impedance of the device is raised. As the voltage exceeds the highest battery voltage, the impedance becomes equal to $R_{0}$ alone, the maximum value. The resulting current versus voltage characteristic is one of constantly increasing slope, as shown in curve $\mathrm{B}$. By combining branches of each type, a characteristic may be achieved whose slope is alternately increasing and decreasing, provided the slope always remains positive. Curve $C$ in Fig. 12 is typical.

The generator of this type which is used with the computer has 22 parallel branches, 11 for positive and 11 for negative voltages. A current-voltage characteristic composed of 22 straight-line segments can be obtained. Such a characteristic can be made to approximate many typical functions with surprisingly good accuracy. Simpler and more special-purpose versions of this device have also been developed, in the form of abrupt current and voltage limiters. These are shown in Fig. 7 together with the Type I generator.

The other arbitrary-function generator (Type II) is based upon a principle developed elsewhere. It utilizes a cathode-ray tube and photocell. The quiescent position of the electron beam is set to one side of the tube and the photocell output is amplified and applied to the plates with a polarity which will drive the beam to the other side. If an opaque template is placed on the surface of the tube, as shown in Fig. 13, the beam spot will be driven below the edge of the template until the light reaching the phototube is just sufficient to produce a signal which will deflect the beam to the edge of the template.

The generator has several limitations which merit discussion. The finite spot size results in some distortion for which compensation is difficult, since distortion depends on the gain of the amplifier, intensity of the spot, the height of the pattern, location of the quiescent position, etc., all of which may vary from time to time. In addition, the halo on the screen contributes an appre- 


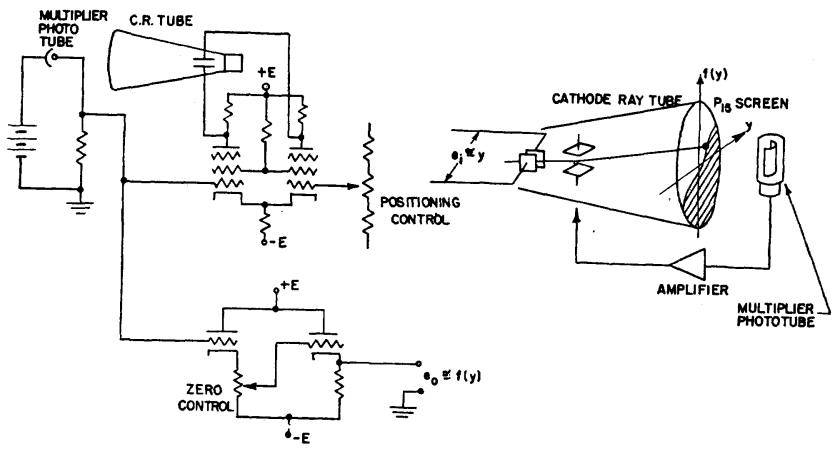

Fig. 13-Schematic diagram of a generator for the arbitrary forcing function of a dependent variable-Type II.

ciable signal which may cause clipping of sharp high peaks of the template, since the halo signal can be picked up on both sides of the peak with the spot completely hidden. A third source of error lies in the parallax resulting from a spot on one side of the cathode-raytube face and a pattern on the other side. Careful proportioning of over-all height and width of the pattern, location of the quiescent position, and adjustment of gain minimize these difficulties, but a definite limitation in accuracy exists. Another limitation lies in the inability of the beam to rise or fall abruptly. Thus, if the dependent variable passes rapidly through a region where the arbitrary function changes abruptly, the output may lag behind the ideal curve. This effect can be minimized by a reduction in vertical scale of the template. Since the time lag is small compared to the period of highest frequency used on this computer, the effect on solutions is usually negligible.

The Type II function generator as developed for the computer is shown in Fig. 7. Its frequency response is best defined in terms of the maximum speed of rise of the cathode-ray beam. Under typical operating conditions, this corresponds to 40 microseconds per inch of deflection along the $f(y)$ axis. This figure was obtained with a P5 phosphor screen having a decay time of approximately 20 microseconds. Screens of this type provide sufficiently fast response characteristics for general use on the computer.

\section{Multiplier}

The multiplication of two arbitrary voltages can be accomplished in many ways. The method which has been adopted for the CIT computer utilizes a doublemodulation and subsequent detection scheme (see the circuit of Fig. 14). A balanced modulator is used to produce sidebands through modulation of a carrier by one of the product terms. The resulting voltage is of the form $E_{1} \cos \left(\omega_{c} t\right)$. This voltage is used as the carrier for a second modulator, which gives a sideband output $E_{1} E_{2} \cos \left(\omega_{c} t\right)$. The carrier voltage is, of course, suppressed in both cases through the use of a balanced modulator. For convenience, a varistor bridge is used for the first, and electron tubes for the second modulator. Detection is accomplished with another variator bridge, minimizing the power-supply requirements of the unit. Isolation between inputs and output is automatically supplied by the high-frequency transformers. It will be noted, however, that the second input voltage is not isolated from system ground. If such isolation is required for both inputs, an additional isolator must be used. Since phase shift of the sidebands results in phase shift of the detected signal, care must be taken to minimize this effect. The first modulator, with untuned transformers, performs very well in this respect. Since tuned circuits are required in the second modulator, for several reasons, the phase shift here is minimized by overcoupling of the transformer in the plate circuit of the modulator.

In the computer, groups of the dc isolators and the multipliers are supplied by a common high-frequency generator, thereby permitting small, compact units.

\section{ACKNOWLEDGMENTS}

Part of the development research on the electronic clmponents of this computer was carried out under a Frederick Gardner Cottrell Research Grant of the Research Corporation. This grant covered research on the multipliers and arbitrary function of the dependent variable. The authors also wish to acknowledge the assistance of R. H. MacNeal and Werner Buchholz, who worked on this project under the Cottrell Research Grant.

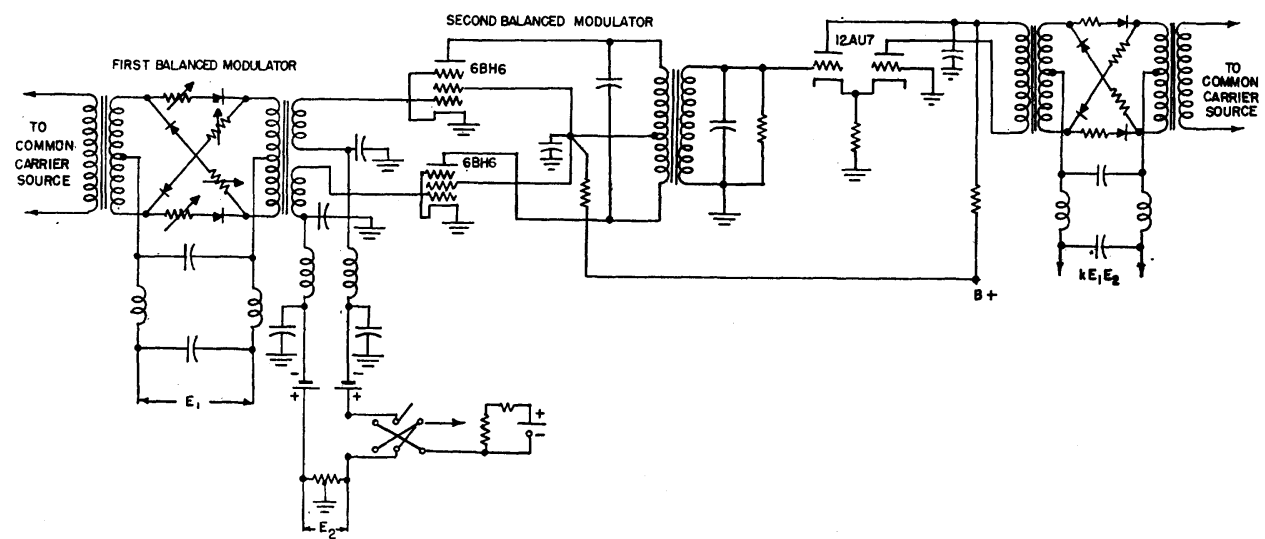

Fig. 14-Schematic diagram of a multiplier. 